\title{
Limits on Purifying Quantum States
}

\section{A new theoretical study identifies fundamental tradeoffs that limit the amount of noise reduction in quantum information systems.}

\author{
By Michael Schirber
}

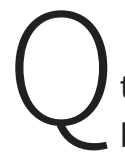
uantum information technology could perform certain tasks faster and more securely than classical methods, but noise is a constant obstacle. Several so-called "purification" schemes exist that can lower the noise, and efforts are ongoing to improve them. But a new study shows that there are fundamental limits on the efficacy of these schemes [1].

To gain a sense of what purification is, imagine that Alice-the quantum-technology poster girl-is computing with entangled photons that are corrupted by environmental interactions. One way that she can purify her input is to generate many copies of the noisy states and then distill a fraction of less noisy states from the larger sample. A typical distillation routine might involve measuring some of the copies to determine which of the unmeasured states have less noise. The more copies generated, the lower the noise can be reduced. However, copies cost time and energy, so Alice has to decide how much purification she can afford.

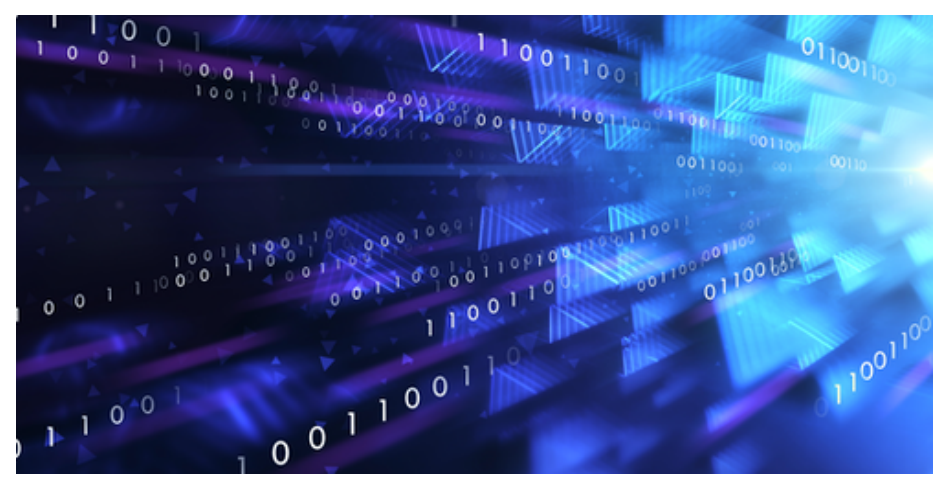

Credit: DKosig/iStock/Getty Images
Kun Fang from the University of Cambridge, UK, and Zi-Wen Liu from the Perimeter Institute for Theoretical Physics, Canada, consider the purification of general kinds of quantum "resources," which could be entanglement, coherence, or some other quantum property. The researchers show that any scheme will be limited by the laws of quantum mechanics, which impose tradeoffs between the desired noise reduction and the efficiency of purification. The authors believe that these fundamental limits shed light on the price of practical quantum technologies while also serving as a benchmark for evaluating a given purification scheme.

This research is published in Physical Review Letters.

Michael Schirber is a Corresponding Editor for Physics based in Lyon, France.

\section{REFERENCES}

1. K. Fang and Z.-W. Liu, "No-go theorems for quantum resource purification,” Phys. Rev. Lett. 125, 060405 (2020). 